\title{
NASH/Liver Fibrosis Prevalence and Incidence of Nonliver Comorbidities among People with NAFLD and Incidence of NAFLD by Metabolic Comorbidities: Lessons from South Korea
}

\author{
Jiyoon Park ${ }^{\mathrm{a}}$ Eunice Yewon Lee ${ }^{\mathrm{b}}$ Jie Lic Mi Jung Jun ${ }^{\mathrm{d}}$ Eileen Yoon ${ }^{\mathrm{e}}$ \\ Sang Bong Ahn ${ }^{f}$ Chuanli Liuc Hongli Yang ${ }^{c}$ Fajuan Rui ${ }^{c}$ Biyao Zou ${ }^{b}$ \\ Linda Henry ${ }^{b}$ Dong Hyun Lee ${ }^{d}$ Dae Won Jung Ramsey C. Cheung ${ }^{b}$ h \\ Mindie H. Nguyen ${ }^{b}$

\begin{abstract}
aDepartment of Internal Medicine, Santa Clara Valley Medical Center, San Jose, CA, USA; ${ }^{b}$ Division of Gastroenterology and Hepatology, Stanford University Medical Center, Palo Alto, CA, USA; 'Department of Infectious Disease, The Provincial Hospital Affiliate of Shandong University, Jian, PR China; ${ }^{d}$ Division of Gastroenterology, Department of Internal Medicine, Good Gang-An Hospital, Busan, South Korea; 'Department of Internal Medicine, Inje University Sanggye Paik Hospital, Seoul, South Korea; fDepartment of Internal Medicine, Nowon Eulji Medical Center, Eulji University College of Medicine, Seoul, South Korea; 9Department of Gastroenterology, Hanyang University, Seoul, South Korea; 'Division of Gastroenterology and Hepatology, Veterans Affairs Palo Alto Health Care System, Palo Alto, CA, USA
\end{abstract}

\section{Keywords}

Fatty liver · Epidemiology · Mortality · Diabetes · Metabolic syndrome - Obesity

\footnotetext{
Abstract

Background: NAFLD incidence, NASH prevalence, NAFLD fibrosis prevalence, incidence of metabolic comorbidities, and mortality data in the NAFLD population remain limited. Aims: We used a meta-analytic approach to "stage" NAFLD among the Korean population. Methods: We searched PubMed, Embase, Cochrane Library, and KoreaMed from inception until June 29, 2019, and calculated pooled estimates via the random-effects model. Results: We screened 1,485 studies and analyzed 191 eligible studies: 179 (3,556,579 participants) for NAFLD prevalence and outcome analysis and 32 (1,089,785 participants) for NAFLD incidence analysis. NAFLD prevalence was $31.46 \%$ overall and $50-60 \%$ in those
}

karger@karger.com www.karger.com/ddi

Karger $\frac{1}{\%}$ BOPEN ACCESS
(C) 2021 The Author(s)

Published by S. Karger AG, Basel

This is an Open Access article licensed under the Creative Commons Attribution-NonCommercial-4.0 International License (CC BY-NC) (http://www.karger.com/Services/OpenAccessLicense), applicable to the online version of the article only. Usage and distribution for commercial purposes requires written permission. with metabolic risks. The incidence (per 1,000 person-years) of NAFLD was 42.8 overall and $70-77 \%$ in those with metabolic risk. The incidence (per 1,000 person-years) of new-onset T2DM, hypertension, cardiovascular disease, and chronic kidney disease was found to be $16.9,47.9,100.6$, and 13.9, respectively. From biopsy data, $30.21 \%$ of the NAFLD population had moderate-to-severe steatosis (9 studies, 2,461 participants) and $52.27 \%$ had NASH (7 studies, 1,168 participants) and $85.41 \%$ had fibrosis <stage 2 ( 8 studies, 1,995 participants). All-cause mortality was 2.6 (1.3 if without malignancy) per 1,000 person-years. Conclusions: The overall prevalence of NAFLD was $31.46 \%$ with an incidence rate of 42.8 per 1,000 person-years. NASH prevalence was $52 \%$ but $<15 \%$ had significant fibrosis. The prevalence and incidence of nonliver comorbidities was high especially for cardiovas-

Jiyoon Park and Eunice Yewon Lee contributed equally.
Correspondence to:

Mindie H. Nguyen, mindiehn@stanford.edu 
cular disease incidence. The burden of NAFLD is high in Korea. Health policy efforts need to be directed towards reversing the course of NAFLD disease. @ 2021 The Author(s).

Published by S. Karger AG, Basel

\section{Introduction}

Nonalcoholic fatty liver disease (NAFLD) is the leading cause of chronic liver disease worldwide and is associated with a variety of medical conditions, including metabolic syndrome, diabetes, and obesity [1-5]. The growing prevalence of NAFLD parallels the obesity epidemic worldwide and in many Asian countries including South Korea [3, 4, 6-8]. Due to South Korea's highly organized and efficient health checkup program, South Korea has been able to provide a large body of epidemiologic literature on various medical conditions, including NAFLD [9-11]. However, due to focused search terms that often include "Asia" or "Korea," recent global and regional meta-analyses have likely missed several relevant studies, since many do not include the region or country name in their titles; for example, one meta-analysis of global NAFLD epidemiology only included 14 total studies for Asia while one meta-analysis of Asia NAFLD epidemiology using more expanded search terms included 237 studies, of which 61 were from Korea [3, 4].

In addition, while liver fibrosis is well known to correlate with mortality and nonliver comorbidities as competing causes of death in people with NAFLD, data on the distribution of nonalcoholic steatohepatitis (NASH) and fibrosis, incidence of nonliver comorbidity development, incidence of NAFLD among subgroups of people with metabolic comorbidities, and cause-specific mortality rates remain scarce globally $[3,4,6,12,13]$. Meanwhile, these data are highly relevant to inform practice, other stakeholders, and future modeling studies to assist public health efforts, and these knowledge gaps should be addressed.

Therefore, using a systematic review and meta-analytic approach focusing on data from South Korea with a broader search strategy that included study author location and a Korean language database, we aimed to not only estimate the prevalence of NAFLD in the general population and in subgroups but also evaluate the prevalence of NASH, advanced fibrosis, and incidence of comorbidities among people with NAFLD as well as estimate the incidence of NAFLD in the general population and in subgroups while also determining mortality outcomes among the NAFLD population.

Prevalence/Incidence of NAFLD, NASH, and Fibrosis

\section{Methods}

We adhered to the PRISMA statement for the conduct of metaanalyses of observational studies (http://www.prisma-statement. $\operatorname{org} /)$.

\section{Search Strategy and Selection Criteria}

We searched PubMed, Embase, Cochrane, and KoreaMed library databases to identify published studies from inception to June 29, 2019, without language restriction. The search strategy is summarized in Figure 1 with additional details in the online supplementary file (see www.karger.com/doi/10.1159/000514953 for all online suppl. material). We excluded studies that did not specify exclusions of other causes of chronic liver disease, such as viral hepatitis and alcoholic liver disease. If studies used the same cohort, we used data from the study with the largest number of participants, longest follow-up, or most comprehensive data.

Study Identification, Data Extraction, and Quality Assessment

Two authors independently performed the literature search and extracted the data using a case report form developed for this study including study characteristics as listed in online supplementary Tables 1 and 2. A quality assessment scale was developed for this study based on the Newcastle-Ottawa scale, comprising 3 domains: selection, comparability, and outcome with a maximum score of 9 . The studies with 7-9 stars were considered low risk of bias, 4-6 stars as moderate risk, and 1-3 stars as high risk. The scores of included studies are shown in online supplementary Table 3.

\section{Study Definitions}

We defined NAFLD as fatty liver identified via ultrasound, CT, serum-based index, or controlled attenuated parameter in the absence of excessive alcohol consumption and other causes of chronic liver disease [10]. However, in NAFLD incidence analysis, only studies that used ultrasound to determine NAFLD diagnosis were included. Normal weight in this study was defined as BMI between 18.5 and $23 \mathrm{~kg} / \mathrm{m}^{2}$, overweight with a BMI between 23 and $25 \mathrm{~kg} /$ $\mathrm{m}^{2}$, and obese with a BMI $\geq 25 \mathrm{~kg} / \mathrm{m}^{2}$ or waist circumference $>90$ $\mathrm{cm}$ in males or $>85 \mathrm{~cm}$ in females [14].

Health checkup center studies use data from health screenings offered by hospitals and clinics across the country, where basic screening is offered for free by the government $[9,10]$. In these checkups, people receive routine health checkups including labs and imaging. Clinical clinic-based studies are studies that use data from regular medical clinics that evaluate both new medical conditions and routine monitoring of chronic medical conditions. Survey studies are those that collect data through community epidemiological survey.

For the purpose of this study, the overall population was defined as studies whose cohort included both sexes, ages 18 years and above, health checkup, and clinical center as well as survey study participants without any restriction on demographics. Studies that included only males or females were not included for the overall analysis but were included in the subgroup analysis looking at either males or females. The overall prevalence analysis included community survey data, health checkup center data, and clinical clinic-based study data. Persons considered as living liver donors were considered well individuals without selected diseases or demographics and thus also included in the overall prevalence analysis. Geographic regions were analyzed by province and city level. 


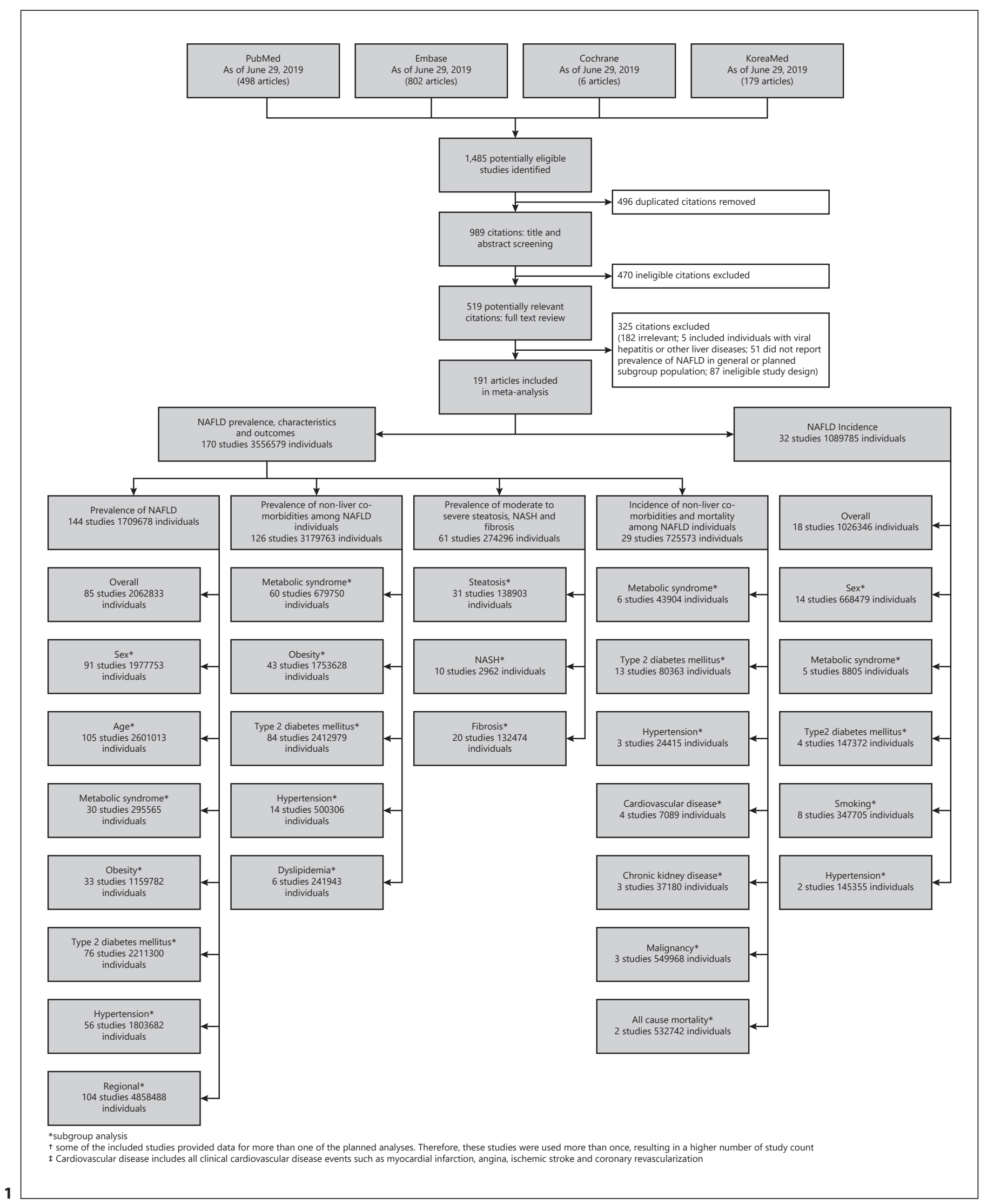

(For legend see next page.) 
Grading of steatosis was as reported by individual studies by either ultrasound or liver biopsy. Generally, steatosis was determined by comparing the echogenicity of the hepatic parenchyma to the renal parenchyma or the hepatic parenchymal attenuation to the spleen parenchyma or determining the posterior attenuation or vessel blurring in studies using ultrasound. For liver biopsy studies, the percentage of hepatocytes showing fatty changes was used to grade steatosis. Fibrosis staging was determined by liver biopsy or elastography and was generally reported by individual studies as per conventional standard. In studies that used elastography to stage fibrosis, fibrosis staging was often reported as aggregated data (fibrosis stage F2 or greater; fibrosis stage F3 or greater); therefore, we presented data from liver biopsies in the same manner for comparison. We also presented the pooled data for individual fibrosis stages using data from liver biopsy studies with 120 participants or more to avoid bias introduced by few numbers of participants for each individual fibrosis stage.

\section{Statistical Analysis}

A random-effects model was used to determine the pooled estimates of each study outcome. The pooled incidence of NAFLD was determined by taking the number of participants who initially did not have NAFLD but developed NAFLD later and calculating the follow-up period (person-years). Heterogeneity was determined using the Cochran Q-statistic and $I^{2}$ statistic. Moderate to severe heterogeneity was defined as a $p$ value of $<0.05$ in Q-statistic and $I^{2} \geq 50 \%$. Subgroup analyses were performed to determine the source of heterogeneity. Meta-regression was performed to determine the variables associated with NAFLD prevalence including median study year, age, obesity, history of hypertension, metabolic syndrome, T2DM, and study region. A funnel plot and Egger's test were used to assess for publication bias. We used the metapackages in $\mathrm{R}$ statistical software (version 3.6.1). This study was performed in accordance with the principles of the Declaration of Helsinki (1964).

\section{Results}

\section{Study Selection and Characteristics}

From the 1,485 studies screened, 191 studies with $4,646,364$ participants were included in study analysis including 13 studies identified only by the KoreaMed database. A total of 179 studies with 3,556,579 individuals were identified for the analyses related to NAFLD prevalence, characteristics of individuals with NAFLD, incidence of nonliver comorbidities, and incidence of mortality among

Fig. 1. Study selection. *Subgroup analysis. ${ }^{\dagger}$ Some of the included studies provided data for more than one of the planned analyses. Therefore, these studies were used more than once, resulting in a higher number of study count. ${ }^{\ddagger}$ Cardiovascular disease includes all clinical cardiovascular disease events such as myocardial infarction, angina, ischemic stroke, and coronary revascularization. NAFLD, nonalcoholic fatty liver disease; NASH, nonalcoholic steatohepatitis.

Prevalence/Incidence of NAFLD, NASH, and Fibrosis the NAFLD population, while 32 studies with $1,089,785$ individuals were identified for analysis of NAFLD incidence (Fig. 1). The included studies were published between 2002 and 2019, with the majority from 2010 or after. The study periods spanned from 1995 to 2017 . Among the 85 studies used for overall prevalence analysis, 1 study used data from clinical centers, 73 studies used data from health checkup centers, 7 studies used survey data, and 4 used liver biopsy data from potential living liver donors with no known liver disease before biopsy. Of the 85 studies included in the overall prevalence analysis, 57 provided stratified data for males and 57 for females and were included in the pooled subanalyses for males and females, respectively. In addition, 31 studies included only males and were added to the pooled analysis for males, while 12 studies were conducted in females only and provided additional data for the female subgroup analysis. In total, the subanalysis for males included 88 studies and the subanalysis for females included 69 studies. Among studies included in the NAFLD incidence analysis, 18 studies provided data for the overall population and all used data from health checkup centers, while 14 studies provided only subgroup data. The characteristics of included studies are shown in online supplementary Tables 1 and 2.

\section{Prevalence of NAFLD in South Korea, Overall and by Diagnostic Methods}

The NAFLD prevalence in the overall population was $31.46 \%$ (95\% CI: 28.61-34.45\%) (85 studies, 2,063,118 participants) (Table 1). The diagnosis of NAFLD was made by ultrasound in 65 studies, fatty liver index (FLI) in 7 studies, hepatic steatosis index (HSI) in 4 studies, controlled attenuated parameter in 4 studies, liver biopsy in 4 studies, and CT in 1 study. The prevalence of NAFLD varied by the diagnostic method, with the highest via liver biopsy at 50.04\% (95\% CI: 42.29-51.68, 4 studies, 4,212 participants) and the lowest with the fatty liver index (FLI) at $12.03 \%$ (95\% CI: 9.59-14.98, 7 studies, 68,892 participants) $(p<0.0001)$. In all studies that used liver biopsy for diagnosis of NAFLD, liver biopsy was taken from all potential liver donors as part of their predonation assessment. In 1 out of 4 studies, only participants without evidence of fatty liver on ultrasound and normal serum aminotransferases had their liver biopsies included in the study [15]. NAFLD in all 4 studies was defined as steatosis $>5 \%$, and a majority of NAFLD found by liver biopsy had steatosis between 5 and $30 \%$. The prevalence of NAFLD was $33.64 \%$ (95\% CI: $31.20-36.16 \%$ ) for ultrasound (65 studies, 1,940,473 participants) and $22.32 \%$ with CT (1 study, 1,886 participants). The study that used 
Table 1. Prevalence of NAFLD in the general population and by subgroup

\begin{tabular}{|c|c|c|c|c|c|}
\hline Population & $\begin{array}{l}\text { Studies, } \\
n\end{array}$ & $\begin{array}{l}\text { Participants, } \\
n\end{array}$ & $\begin{array}{l}\text { Prevalence of NAFLD } \\
(95 \% \text { CI }), \%\end{array}$ & $I^{2}, \%$ & $p$ value \\
\hline General population & 85 & $2,063,118$ & $31.46(28.61-34.45)$ & 99.9 & \\
\hline \multicolumn{6}{|l|}{ Method of diagnosis } \\
\hline Ultrasound & 65 & $1,940,473$ & $33.64(31.20-36.16)$ & 99.9 & \multirow{6}{*}{$<0.0001$} \\
\hline CT scan & 1 & 1,886 & $22.32(20.50-24.26)$ & 0 & \\
\hline CAP & 4 & 5,876 & $45.92(42.22-51.68)$ & 89.6 & \\
\hline Liver biopsy & 4 & 4,212 & $50.04(42.29-57.79)$ & 95.8 & \\
\hline Fatty liver index (FLI) & 7 & 68,892 & $12.03(9.59-14.98)$ & 99.1 & \\
\hline Hepatic steatosis index (HSI) & 4 & 41,799 & $17.90(10.09-29.74)$ & 99.8 & \\
\hline \multicolumn{6}{|l|}{ Sex } \\
\hline Male & 88 & $1,370,855$ & $39.83(37.82-41.89)$ & 99.8 & \multirow{2}{*}{$<0.0001$} \\
\hline Female & 69 & 635,022 & $23.27(21.29-25.38)$ & 99.5 & \\
\hline \multicolumn{6}{|l|}{ Age } \\
\hline $30-39$ & 16 & $1,459,501$ & $30.56(26.06-35.45)$ & 100 & \multirow{4}{*}{0.0037} \\
\hline $40-49$ & 61 & $1,067,623$ & $30.52(28.25-32.89)$ & 99.8 & \\
\hline $50-59$ & 38 & 155,881 & $36.12(32.81-39.56)$ & 99.4 & \\
\hline$>60$ & 6 & 4,432 & $33.82(33.82-46.97)$ & 94.5 & \\
\hline \multicolumn{6}{|l|}{ Metabolic syndrome } \\
\hline Metabolic syndrome & 34 & 61,653 & $63.20(59.72-66.55)$ & 98.5 & \multirow{2}{*}{$<0.0001$} \\
\hline No metabolic syndrome & 36 & 293,466 & $24.22(21.65-26.98)$ & 99.6 & \\
\hline \multicolumn{6}{|l|}{ Obesity } \\
\hline Obesity & 33 & 413,480 & $56.70(52.09-61.20)$ & 99.9 & \multirow{2}{*}{$<0.0001$} \\
\hline No obesity & 38 & 814,945 & $19.52(16.52-22.91)$ & 99.9 & \\
\hline \multicolumn{6}{|l|}{ Type 2 diabetes mellitus } \\
\hline Type 2 diabetes mellitus & 58 & 94,055 & $58.18(54.65-61.62)$ & 98.8 & \multirow{2}{*}{$<0.0001$} \\
\hline No type 2 diabetes mellitus & 85 & $2,140,089$ & $32.25(30.44-34.11)$ & 99.9 & \\
\hline \multicolumn{6}{|l|}{ Hypertension } \\
\hline Hypertension & 57 & 263,781 & $47.00(44.35-49.66)$ & 99.4 & \multirow{2}{*}{$<0.0001$} \\
\hline No hypertension & 65 & $1,561,997$ & $30.23(28.07-32.48)$ & 99.9 & \\
\hline \multicolumn{6}{|l|}{ Metabolic syndrome and obesity } \\
\hline No metabolic syndrome and no obesity & 5 & 26,101 & $18.02(11.93-26.30)$ & 99.2 & \multirow{4}{*}{$<0.0001$} \\
\hline Metabolic syndrome and no obesity & 5 & 11,876 & $46.75(41.19-52.39)$ & 95.9 & \\
\hline No metabolic syndrome and obesity & 5 & 6,589 & $54.76(47.12-62.19)$ & 95.6 & \\
\hline Metabolic syndrome and obesity & 5 & 10,434 & $80.99(74.82-85.93)$ & 97.2 & \\
\hline
\end{tabular}

References for included studies are provided in online supplementary Table 1. Subgroup analysis may include studies that only provide subgroup data, and thus subgroup sample size may be larger than the overall cohort. NAFLD, nonalcoholic fatty liver disease; CAP, controlled attenuation parameter.

CT to diagnose NAFLD had more females in the study cohort than males [16]. Because the majority of studies diagnosed NAFLD by ultrasound, which is also the most commonly used modality for diagnosis of fatty liver in routine practice, we elected to use only those studies that used ultrasound (unless specified) for subsequent characterization of NAFLD.

\section{By Sex, Age, and Obesity}

Males had a higher NAFLD prevalence compared to females (39.84 vs. $22.42 \%, p<0.0001$ ) (Table 1 ). The prevalence of NAFLD increased with age, with the lowest at
$28.46 \%$ in the 30 - to 39 -year-old group and the highest at $36.84 \%$ for the $>60$-year-old group $(p=0.0035)$ (Table 1 ). NAFLD prevalence was higher among obese individuals compared to nonobese individuals (55.23 vs. $20.01 \%, p<$ 0.0001 ) (Table 1).

\section{By Metabolic Risk Factors}

The prevalence of NAFLD was statistically higher in individuals with metabolic syndrome (63.55 vs. $24.58 \%$, $p \leq 0.0001$ ), T2DM (57.78 vs. $31.61 \%, p<0.0001$ ), or hypertension ( 46.96 vs. $29.70 \%, p<0.0001$ ) compared to those without (Table 1). There were 5 studies that exam- 
Table 2. Prevalence of nonliver comorbidities by the presence of NAFLD

\begin{tabular}{|c|c|c|c|c|}
\hline Population & $\begin{array}{l}\text { Studies, } \\
n\end{array}$ & $\begin{array}{l}\text { Participants, } \\
n\end{array}$ & $\begin{array}{l}\text { Prevalence of nonliver } \\
\text { comorbidities } \\
(95 \% \text { CI }), \%\end{array}$ & $p$ value \\
\hline \multicolumn{5}{|c|}{ Metabolic syndrome } \\
\hline NAFLD $^{a}$ & 42 & 111,277 & $40.17(33.70-47.01)$ & \multirow{2}{*}{$<0.0001$} \\
\hline No NAFLD ${ }^{a}$ & 41 & 249,315 & $11.16(8.24-14.95)$ & \\
\hline \multicolumn{5}{|l|}{ Obesity } \\
\hline NAFLD $^{\mathrm{a}}$ & 35 & 337,086 & $61.75(59.05-64.39)$ & \multirow{2}{*}{$<0.0001$} \\
\hline No NAFLD ${ }^{\mathrm{a}}$ & 35 & 966,790 & $21.51(17.85-25.69)$ & \\
\hline \multicolumn{5}{|c|}{ Type 2 diabetes mellitus } \\
\hline NAFLD $^{\mathrm{a}}$ & 61 & 515,616 & $14.23(11.68-17.23)$ & \multirow{2}{*}{$<0.0001$} \\
\hline No NAFLD ${ }^{\mathrm{a}}$ & 59 & $1,280,636$ & $5.23(4.20-6.49)$ & \\
\hline \multicolumn{5}{|l|}{ Hypertension } \\
\hline NAFLD ${ }^{a}$ & 13 & 92,594 & $23.98(17.44-32.02)$ & \multirow{2}{*}{0.31} \\
\hline No NAFLD ${ }^{\mathrm{a}}$ & 13 & 266,102 & $17.23(11.98-24.16)$ & \\
\hline \multicolumn{5}{|l|}{ Dyslipidemia } \\
\hline NAFLD $^{\mathrm{a}}$ & 3 & 16,383 & $38.58(5.47-87.21)$ & \multirow{2}{*}{0.66} \\
\hline No NAFLD & 3 & 30,138 & $15.60(3.15-51.24)$ & \\
\hline
\end{tabular}

Italic values emphasize the subgroup with NAFLD. References for included studies are provided in online supplementary Table 1 . NAFLD, nonalcoholic fatty liver disease. ${ }^{\text {a }} I^{2}>98.4$.

ined the relative contribution of metabolic syndrome and obesity. Individuals with obesity and metabolic syndrome $(N=10,434)$ had a higher prevalence of NAFLD $(80.99 \%$, 95\% CI: 74.82-85.93\%), followed by individuals with obesity but without metabolic syndrome $(N=6,589$; $54.76 \%$, 95\% CI: 47.12-62.19\%) and by those with metabolic syndrome without obesity $(N=11,876 ; 46.75 \%, 95 \%$ CI: 41.19-52.39\%), and lowest among those with neither metabolic syndrome nor obesity $(N=26,101 ; 18.02 \%$, 95\% CI: 11.93-26.30\%) $(p<0.001)($ Table 1$)$.

\section{By Region}

Only one study specifically determined the countrywide prevalence of NAFLD and reported an overall prevalence of $34.23 \%$ (95\% CI: 33.23-34.86\%). By province, Gyeonggi-do had a higher NAFLD prevalence compared to Gyeongsang-do ( 33.10 vs. $18.19 \%, p=0.008$ ) (online suppl. Table $4 \mathrm{a})$. We were unable to identify studies that provided data for other provinces. Comparing Seoul versus outside of Seoul, the prevalence of NAFLD was significantly higher in Seoul (34.55 vs. $25.22 \%, p=0.01$ ) (online suppl. Table $4 \mathrm{~b}$ ).

\section{Characteristics of Individuals with NAFLD}

Prevalence of Nonliver Comorbidities

In our analysis, the prevalence of metabolic syndrome in individuals with NAFLD was $40.17 \%$ (95\% CI: 33.70
47.01\%), compared to those who did not have NAFLD (11.16\%, 95\% CI: 8.24-14.95\%) ( $p<0.0001)$ (Table 2). The prevalence of obesity in individuals with NAFLD was 61.75\% (95\% CI: 59.05-64.39\%) (Table 2), compared to the group without NAFLD (21.51\%, 95\% CI: $17.85-$ $25.69 \%)(p<0.0001)$. In individuals with NAFLD, the prevalence of T2DM was $14.23 \%$ (95\% CI: $11.68-17.23 \%)$ compared to the group without NAFLD $(5.23 \%, 95 \%$ CI: $4.20-6.49 \%)(p<0.0001)$. Unlike metabolic syndrome, T2DM, and obesity, there was no statistically significant difference in the prevalence of hypertension $(p=0.33)$ or dyslipidemia ( $p=0.66$ ) between the NAFLD and nonNAFLD groups.

Prevalence of Moderate-to-Severe Hepatic Steatosis, Fibrosis, and NASH

Among participants with NAFLD, 26.50\% (95\% CI: 20.79-33.12) had moderate-to-severe grade steatosis, which did not differ significantly between ultrasound and biopsy-based studies ( 25.20 vs. $30.21 \%, p=0.53$ ) (Table 3). Among those with known NAFLD who underwent liver biopsy for further evaluation, the prevalence of NASH was $52.27 \%$ (95\% CI: 38.13-66.07\%) (Table 3). On the other hand, the prevalence of NASH among the 3 studies from potential liver donors was $0.03 \%$ (95\% CI: 0.03-0.03). 
Table 3. Prevalence of moderate-to-severe steatosis, biopsy-proven NASH, and aggregate fibrosis stages in individuals with NAFLD

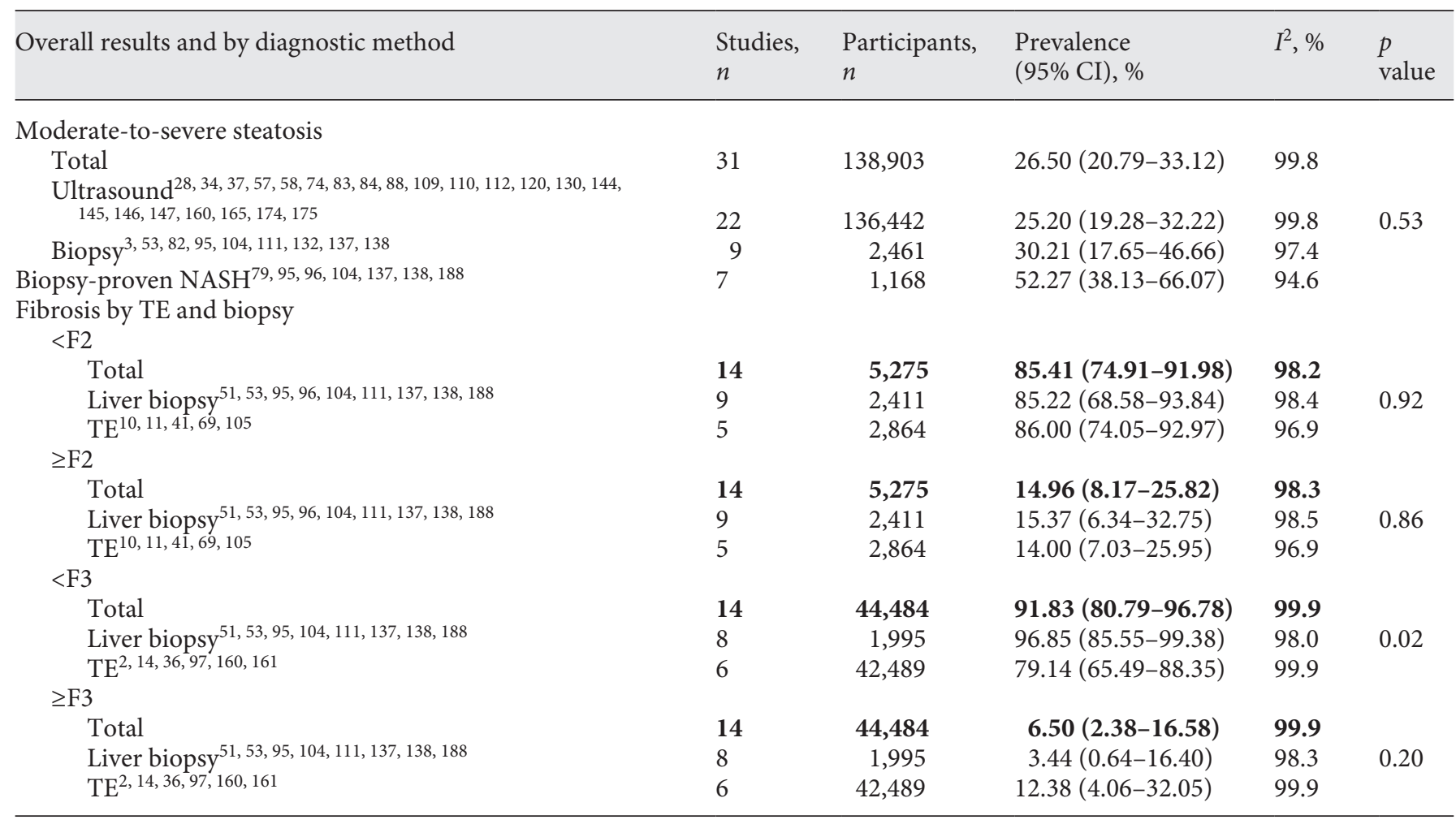

Bold values emphasize total values of steatosis, biopsy-proven NASH, and fibrosis stages. NAFLD, nonalcoholic fatty liver disease; $\mathrm{NASH}$, nonalcoholic steatohepatitis; TE, transient elastography.

Table 4. Prevalence of individual fibrosis stages in individuals with NAFLD

\begin{tabular}{lllll}
\hline Overall results and by diagnostic method & $\begin{array}{l}\text { Studies, } \\
n\end{array}$ & $\begin{array}{l}\text { Participants, } \\
n\end{array}$ & $\begin{array}{l}\text { Prevalence } \\
(95 \% \text { CI }), \%\end{array}$ & $I^{2}, \%$ \\
\hline $\begin{array}{l}\text { F0 Liver biopsy } 51,53,104,111,188 \\
\text { F1 Liver biopsy } 51,53,104,111,188\end{array}$ & 5 & 1,805 & $74.19(37.06-93.35)$ & 99.2 \\
F2 Liver biopsy $51,53,104,111,188$ & 5 & 1,805 & $11.02(3.36-30.66)$ & 98.4 \\
F3 Liver biopsy $51,53,104,111,188$ & 5 & 1,805 & $4.77(1.71-12.60)$ & 95.7 \\
$\begin{array}{l}\text { F4 (cirrhosis) } \\
\text { Liver biopsy } 51,53,104,111,188\end{array}$ & 5 & 1,805 & $2.00(0.46-8.23)$ & 95.5 \\
\hline $\begin{array}{l}\text { NAFLD, nonalcoholic fatty liver disease. } \\
\quad\end{array}$ & 5 & 1,805 & $0.77(0.05-11.35)$ & 98.3 \\
\hline
\end{tabular}

Liver fibrosis data were available from 14 studies and 5,275 participants - 5 studies with 2,864 participants used transient elastography (TE) to assess for hepatic fibrosis and 9 studies with 2,411 participants used liver biopsy data to determine fibrosis (Table 3). Among those with NAFLD, the prevalence of fibrosis stage F2 or greater was $14.96 \%$ (95\% CI: $8.17-25.82$ ) and $<$ F2 was $85.41 \%$ (95\% CI: 74.91-91.98); fibrosis stage F3 or greater was $6.50 \%$ 
Table 5. The incidence of nonliver comorbidity and the incidence of mortality by the presence of NAFLD

\begin{tabular}{|c|c|c|c|c|c|}
\hline & $\begin{array}{l}\text { Studies, } \\
n\end{array}$ & $\begin{array}{l}\text { Participants, } \\
n\end{array}$ & $\begin{array}{l}\text { Incidence of comorbidity (per } \\
1,000 \text { person-years, } 95 \% \mathrm{CI} \text { ) }\end{array}$ & $I^{2}, \%$ & $p$ value \\
\hline \multicolumn{6}{|c|}{ Metabolic syndrome } \\
\hline NAFLD & 3 & 12,730 & $122.3(56.2-213.8)$ & 99.8 & \multirow{2}{*}{0.26} \\
\hline No NAFLD & 3 & 31,174 & $45.9(0.0-182.3)$ & 100 & \\
\hline \multicolumn{6}{|c|}{ Type 2 diabetes mellitus } \\
\hline NAFLD & 12 & 61,213 & $16.9(12.0-22.6)$ & 99.0 & \multirow{2}{*}{0.0001} \\
\hline No NAFLD & 13 & 182,708 & $5.2(2.6-8.8)$ & 99.7 & \\
\hline \multicolumn{6}{|l|}{ Hypertension } \\
\hline NAFLD & 3 & 10,644 & $47.9(19.8-88.1)$ & 99.4 & \multirow{2}{*}{0.31} \\
\hline No NAFLD & 3 & 24,415 & $28.2(10.4-54.5)$ & 99.7 & \\
\hline \multicolumn{6}{|c|}{ Cardiovascular disease } \\
\hline NAFLD & 4 & 5,389 & $100.6(53.6-162.2)$ & 99.0 & \multirow{2}{*}{0.49} \\
\hline No NAFLD & 4 & 7,089 & $75.2(34.1-132.3)$ & 99.2 & \\
\hline \multicolumn{6}{|c|}{ Chronic kidney disease } \\
\hline NAFLD & 3 & 17,357 & $13.9(4.4-28.7)$ & 99.0 & \multirow[b]{2}{*}{0.42} \\
\hline No NAFLD & 3 & 37,180 & $8.2(2.2-18.1)$ & 99.6 & \\
\hline \multicolumn{6}{|c|}{ All-cause mortality } \\
\hline NAFLD & 3 & 183,012 & $2.6(1.1-4.8)$ & 99.6 & \multirow{2}{*}{0.54} \\
\hline No NAFLD & 3 & 549,968 & $2.0(1.0-3.3)$ & 99.8 & \\
\hline \multicolumn{6}{|c|}{ All-cause mortality without malignancy } \\
\hline NAFLD & 2 & 174,291 & $1.3(0.9-1.8)$ & 97.8 & \multirow{2}{*}{0.29} \\
\hline No NAFLD & 2 & 532,742 & $1.0(0.7-1.3)$ & 98.7 & \\
\hline
\end{tabular}

Italic values emphasize the subgroup with NAFLD. References for included studies are provided in online supplementary Table 1. NAFLD, nonalcoholic fatty liver disease.

(95\% CI: $2.38-16.58)$ and $<$ F3 was $91.83 \%(95 \% \mathrm{CI}$ : 80.79-96.78). There was no significant difference between those diagnosed with liver biopsy and TE (Table 3). In analysis for pooled estimates of individual fibrosis stage using data from 5 studies (1,805 participants) with a sample size of at least 120 participants each (Table 4), we found that $74.19 \%$ had fibrosis stage F0 (95\% CI: 37.06-93.35), 11.02\% had fibrosis stage F1 (95\% CI: 3.36$30.66), 4.77 \%$ had fibrosis stage F2 (95\% CI: 1.71-12.60), $2.00 \%$ had fibrosis stage F3 (95\% CI: $0.46-8.23$ ), and $0.77 \%$ had fibrosis stage F4 (cirrhosis) (95\% CI: $0.05-$ 11.35).

\section{Incidence of Nonliver Comorbidities among} Individuals with NAFLD

Among the NAFLD population, the incidence per 1,000 person-years was 122.3 (95\% CI: $56.2-213.8$ ) for metabolic syndrome, 47.9 (95\% CI: 19.8-88.1) for hypertension, 100.6 (95\% CI: 53.6-162.2) for cardiovascular disease, 13.9 (95\% CI: 4.4-28.7) for chronic kidney disease, and 16.9 (95\% CI: 12.0-22.6) for T2DM. It is interesting that of the above comorbidities, only T2DM had developed at a significantly higher rate in individuals with NAFLD compared to those without (16.9 vs. 5.2 per 1,000 person-year, 95\% CI: 2.6-8.8) $(p=0.0001)$ (Table 5).

Incidence of Mortality among Individuals with NAFLD

The incidence of all-cause mortality in individuals with NAFLD (6 studies, 732,980 participants) was 2.6 per 1,000 person-years (95\% CI: 1.1-4.8). The incidence of all-cause but noncancer mortality in participants with NAFLD was 1.3 per 1,000 person-years ( $95 \%$ CI: $0.9-1.8$ ) (Table 5). Neither of these 2 mortality rates were statistically different between participants with NAFLD and those without NAFLD $(p=0.29)$.

Incidence of NAFLD, Overall and in Subgroup

Of the study population without NAFLD at baseline by ultrasound, the incidence of NAFLD diagnosed by ultrasound was 42.8 per 1,000 person-years overall (95\% CI: 38.6-47.1 per 1,000 person-years) (Table 6) and higher in males compared to females (50.6 vs. 19.8 per 1,000 person-years, $p<0.0001)$. Individuals with metabolic syn- 
Table 6. The incidence of NAFLD overall and by subgroup

\begin{tabular}{|c|c|c|c|c|c|}
\hline & $\begin{array}{l}\text { Studies, } \\
n\end{array}$ & $\begin{array}{l}\text { Participants, } \\
n\end{array}$ & $\begin{array}{l}\text { Incidence } \\
\text { (per } 1,000 \text { person-years, } 95 \% \mathrm{CI} \text { ) }\end{array}$ & $I^{2}, \%$ & $p$ value \\
\hline Overall incidence & 18 & $1,026,346$ & $42.8(38.6-47.1)$ & 99.7 & \\
\hline \multicolumn{6}{|l|}{ By sex } \\
\hline Male & 14 & 306,501 & $50.6(40.9-61.3)$ & 99.9 & \multirow{2}{*}{$<0.0001$} \\
\hline Female & 12 & 356,978 & $19.8(16.4-23.6)$ & 99.7 & \\
\hline \multicolumn{6}{|c|}{ Metabolic syndrome } \\
\hline Yes & 5 & 1,059 & $77.1(63.9-91.4)$ & 51.2 & \multirow{2}{*}{$<0.0001$} \\
\hline No & 5 & 7,746 & $43.5(34.5-53.5)$ & 93.3 & \\
\hline \multicolumn{6}{|c|}{ Type 2 diabetes mellitus } \\
\hline Yes & 4 & 1,838 & $70.9(65.3-76.7)$ & 0.0 & \multirow{2}{*}{$<0.0001$} \\
\hline No & 4 & 145,534 & $45.1(43.2-47.0)$ & 60.4 & \\
\hline \multicolumn{6}{|l|}{ Smoking } \\
\hline Yes & 8 & 88,951 & $57.5(52.1-63.2)$ & 97.9 & \multirow{2}{*}{$<0.0001$} \\
\hline No & 8 & 258,754 & $32.5(29.2-36.1)$ & 99.0 & \\
\hline \multicolumn{6}{|l|}{ Hypertension } \\
\hline Yes & 2 & 12,590 & $71.0(68.9-73.2)$ & 0.0 & \multirow{2}{*}{$<0.0001$} \\
\hline No & 2 & 132,765 & $42.7(40.3-45.2)$ & 85.1 & \\
\hline
\end{tabular}

Italic values emphasize those subgroups with certain risk factors of NAFLD. References for included studies are provided in online supplementary Table 2. NAFLD, nonalcoholic fatty liver disease.

drome (77.1 vs. 43.5 per 1,000 person-years, $p<0.0001)$ or components of metabolic syndrome had higher NAFLD incidence (per 1,000 person-years) compared to those who did not (obesity 78.0 vs. $40.5, p<0.0001$; T2DM 70.9 vs. $45.1, p<0.0001$; or hypertension 71.0 vs. 42.7 , $p<0.0001$ ) (Table 6). Those with a history of smoking also had higher NAFLD incidence than nonsmokers (57.5 vs. 32.5 per 1,000 person-years, $p<0.0001$ ) (Table 6 ).

\section{Sensitivity Analysis and Publication Bias}

We performed sensitivity analysis for the prevalence of NAFLD in the overall population by excluding studies with quality assessment scores $<7$ points, studies with a sample size $<1,000$, nonclinical-based studies, nonpopulation-based studies, and studies with extremity high $(>50 \%)$ and low $(<10 \%)$ prevalence. All analyses showed similar results as those of the main analysis (online suppl. Tables 5, 6).

Publication bias was found in the analysis of NAFLD prevalence for the overall population $(p=0.039)$. However, after excluding studies of lesser quality (quality assessment scores $<7)$ or smaller sample size $(<1,000$ persons), there were no longer statistically significant biases ( $p=0.55$ and 0.08 , respectively) (online suppl. Table 5; online suppl. Fig. 1).

\section{Discussion}

In this meta-analysis, we aimed to provide a myriad of results that can be used in health policy makers' and other stakeholders' decision-making when creating health and wellness goals for their country. First, we found that the prevalence of NAFLD in South Korea was 31.46\%, though it ranged from a low of $12.3 \%$ when diagnosed by FLI to a high of $50 \%$ when diagnosed with a liver biopsy and $34 \%$ when diagnosed by ultrasound. The prevalence of $31.46 \%$ is similar to the reported rate for the USA (32.3\%) but higher than the global rate of $25 \%$ and that of Asia $(29.6 \%)[3,4,17,18]$. This higher rate could be driven by the fact that most of the studies were from Seoul, where, as seen in China, there is an NAFLD prevalence gradient, where the prevalence was higher in the more urban area of Seoul (34.55\%) compared to the rural area outside of Seoul (25.22\%). This gradient suggests that as the diet and environment become more westernized, the increase in NAFLD may follow [19].

Secondly, we found that males and those with certain comorbidities had a higher prevalence of NAFLD. In fact, males experienced almost twice the prevalence of NAFLD compared to females, and individuals with metabolic syndrome and obesity incurred an NAFLD prevalence of $80 \%$ compared to an NAFLD prevalence of $18 \%$ for those who did not have metabolic syndrome or obesity. 
The overall incidence of NAFLD was 42.8 per 1,000 person-years; however, the NAFLD incidence rate varied according to the presence of certain comorbidities. Those who had T2DM, metabolic syndrome, or hypertension experienced an incidence rate of NAFLD $>70$ cases per 1,000 person-years. The incidence of cardiovascular disease in those with NAFLD was high at 100.6 per 1,000 person-years. In addition, we also noted that individuals with NAFLD had a much higher risk of developing T2DM compared to non-NAFLD as has been reported in other studies [20].

Thirdly, we found that over $50 \%$ of individuals with NAFLD had NASH (diagnosed by liver biopsy). We do acknowledge that there is likely to be a selection bias in these studies, as a liver biopsy is not routinely done in individuals with NAFLD unless there is concern for advanced liver disease; therefore, these rates may be higher than generally found. However, our findings are consistent with another study which reported the pooled NASH prevalence among biopsied individuals from Asia with NAFLD was $>50 \%$ [4]. Fortunately, only $15 \%$ of patients were found to have stage F2 or higher and only about $6 \%$ had stage F3 or higher. Finally, we determined that the mortality rate of individuals with NAFLD in South Korea was lower than the rate in Asia and countries such as China and Hong Kong but higher than that reported from Japan [3].

Together, these findings suggest that the burden of NAFLD and metabolic comorbidity burden of the NAFLD population is high in South Korea, and as such, efforts are needed to address these issues. For example, cardiovascular disease is the number one cause of death among those with NAFLD [21-23], and, as noted in this study, the incidence of cardiovascular disease was quite high [24]. Therefore, efforts such as following the American Heart Association Simple 7 guideline may prove beneficial in managing the risk factors associated with cardiovascular disease in those with NAFLD. In addition, as noted in the recent guidelines from the American Gastroenterology Association, a Mediterranean style diet may also be beneficial in addressing the high rate of NAFLD development among those with obesity, metabolic syndrome, and T2DM [25].

Staging of fibrosis in those with NAFLD is important as it is associated with mortality in those with NAFLD [26]. Though only $15 \%$ of persons in the study were found to have significant fibrosis, over $50 \%$ were found to have $\mathrm{NASH}$, which is the more progressive form of NAFLD and the stage most likely to progress to fibrosis especially in the presence of T2DM [27-30]. Therefore, practitioners should actively monitor for the development of fi- brosis in the patients at the highest risk [31]. The use of noninvasive testing to determine the presence of fibrosis is expanding, but currently the most commonly used and easily calculated test is the fibrosis 4 (FIB-4) score $[32,33]$. However, all noninvasive tests at this time have a gray zone which hampers their use [17]. As such, TE is becoming more popular in the larger medical centers, and, as noted in the study, the prevalence of fibrosis determined by TE was similar to that reported by liver biopsy which helps support the use of TE in the future [34].

Finally, our findings suggest that in Asia there are ethnic, lifestyle, economic, and urbanization variations within Asia $[3,19]$, making it difficult for countries to fully understand its disease burden. This emphasizes the need to estimate the prevalence, incidence, and outcomes of NAFLD by each country and geographic region, supporting further public health measures.

Limitations of this study include the overrepresentation of studies from Seoul. While the Seoul metropolitan area is the leading population center of South Korea (about 10 million), its population may not be representative of the entire country population of about 51 million [35]. Our study population may also include foreign nationals or those residing outside of Korea; however, foreign nationals occupied only $2.9 \%$ of the South Korean population as of 2017, so the vast majority of our study patients are likely South Korean nationals and residents [36]. Furthermore, most studies were done using individuals who were provided free health checkups by large employers in larger hospitals, and thus citizens seeking care at smaller centers may be underrepresented in the analysis $[9,10]$. In addition, though we did not find a statistical difference between the incidence of cardiovascular disease between those with and without NAFLD, the incidence rate was nonetheless very high for those with NAFLD. Reasons for not being able to determine a statistical difference may be due to lack of data separating the severity of cardiovascular disease and data stratifying cardiovascular outcomes by levels of fibrosis which can be the determining factors for some of these associations $[22,30,37,38]$. Within the analyses that used liver biopsy as a method of staging and/or diagnosis, the reported results can be biased by the inherent biases of liver biopsy which include different interpretations of the liver histology by the pathologist and provider-dependent variations. In addition, those individuals who had liver biopsies might have worse disease due to selection and surveillance bias. Therefore, we were selective in the analysis that included liver biopsies and carefully considered the populations the biopsies came from (liver donors vs. in- 
dividuals with NAFLD). Another limitation is the high heterogeneity found among studies. However, this is a common problem among meta-analyses of pooled proportions and especially of this size, which we could not explain through our subanalyses [39-41]. A broader search that was required to best analyze topics with limited data such as NASH prevalence and incidence of mortality in the NAFLD population may also add high heterogeneity. Due to lack of data, we also were not able to provide cardiovascular- and liver-related mortality. Given the heterogeneity of using a meta-analytic approach, we recommend more studies be conducted investigating cause-specific mortality.

Nevertheless, despite these limitations, this meta-analysis which pooled data from almost 200 studies with over 4 million participants helps fill the knowledge gaps in the prevalence of NASH/fibrosis and incidence of nonliver comorbidities and mortality among people with NAFLD, in addition to providing country-specific data for South Korea.

In conclusion, about one-third of the South Korean population is affected by NAFLD, and those with NAFLD experience higher prevalence of metabolic syndrome, obesity, and T2DM than individuals without NAFLD. These data demonstrate that individuals with NAFLD require complicated medical management necessitating knowledge of multiple chronic disorders. As there is no pharmacological cure for NAFLD at this time, early detection and prevention of the disease is key to reducing the clinical and economic burden of the disease [42]. Therefore, policy makers need to focus efforts on interventions such as how to eat healthier and how to improve environment to promote healthy living.

\section{Acknowledgement}

The authors specially acknowledge www.mapchart.net for the generous sharing of map tools used to generate all of the maps in this article.

\section{Statement of Ethics}

We adhered to the PRISMA statement for the conduct of metaanalyses of observational studies (http://www.prisma-statement. org/). This study was performed in accordance with the principles of the Declaration of Helsinki (1964).

\section{Conflict of Interest Statement}

R.C.: research support (Gilead). M.H.N.: research support (Enanta, Gilead, and Pfizer) and consultant and/or an advisory board member (Intercept and Gilead). All other authors have nothing to disclose.

\section{Funding Sources}

No external funding to disclose.

\section{Author Contributions}

Study concept and study supervision: M.H.N. Study design: D.H.L., J.P., and M.H.N. Data analysis: E.Y.L., B.Z., J.P., and M.H.N. Drafting of the manuscript: J.P., L.H., R.C., and M.H.N. Data collection: E.Y.L., D.H.L., J.L., F.R., J.J.M., C.L., and H.Y. Data interpretation and critical review/revision of the manuscript: all authors.

\section{References}

1 Pagano G, Pacini G, Musso G, Gambino R, Mecca F, Depetris N, et al. Nonalcoholic steatohepatitis, insulin resistance, and metabolic syndrome: further evidence for an etiologic association. Hepatology. 2002;35(2):367-72.

2 Lee K, Sung JA, Kim JS, Park TJ. The roles of obesity and gender on the relationship between metabolic risk factors and non-alcoholic fatty liver disease in Koreans. Diabetes Metab Res Rev. 2009;25(2):150-5.

3 Li J, Zou B, Yeo YH, Feng Y, Xie X, Lee DH, et al. Prevalence, incidence, and outcome of non-alcoholic fatty liver disease in Asia, 1999-2019: a systematic review and metaanalysis. Lancet Gastroenterol Hepatol. 2019; 4(5):389-98.
4 Younossi ZM, Koenig AB, Abdelatif D, Fazel Y, Henry L, Wymer M. Global epidemiology of nonalcoholic fatty liver disease-meta-analytic assessment of prevalence, incidence, and outcomes. Hepatology. 2016;64(1):73-84.

5 Goldberg D, Ditah IC, Saeian K, Lalehzari M, Aronsohn A, Gorospe EC, et al. Changes in the prevalence of hepatitis $\mathrm{C}$ virus infection, nonalcoholic steatohepatitis, and alcoholic liver disease among patients with cirrhosis or liver failure on the waitlist for liver transplantation. Gastroenterology. 2017;152(5):10909.e1.

6 Korean Association for the Study of the Liver (KASL). KASL clinical practice guidelines: management of nonalcoholic fatty liver disease. Clin Mol Hepatol. 2013;19(4):325-48.

7 Liver Disease White Paper 2013. Korean Association for the Study of the Liver. 2013.
8 Anstee QM, Targher G, Day CP. Progression of NAFLD to diabetes mellitus, cardiovascular disease or cirrhosis. Nat Rev Gastroenterol Hepatol. 2013;10(6):330-44.

9 Overview of National Health Insurance Danuri [updated April 2019]. Available from: http: //www.liveinkorea.kr/portal/USA/page/ contents.do? menuSeq $=3722$ \&pageSeq $=43$.

10 Seo MH, Lee W-Y, Kim SS, Kang J-H, Kang J-H, Kim KK, et al. 2018 Korean Society for the study of obesity guideline for the management of obesity in Korea. J Obes Metab Syndr. 2019;28(1):40-5.

11 Kim HS, Shin DW, Lee WC, Kim YT, Cho B. National screening program for transitional ages in Korea: a new screening for strengthening primary prevention and follow-up care. J Korean Med Sci. 2012;27(Suppl):S70-5. 
12 Younossi ZM. The epidemiology of nonalcoholic steatohepatitis. Clin Liver Dis. 2018; 11(4):92-4.

13 Estes C, Chan HLY, Chien RN, Chuang WL, Fung J, Goh GB, et al. Modelling NAFLD disease burden in four Asian regions-2019-2030. Aliment Pharmacol Ther. 2020 Apr;51(8): 801-11.

14 Appropriate body-mass index for Asian populations and its implications for policy and intervention strategies. Lancet. 2004; 363(9403): 157-63.

15 Ahn JS, Sinn DH, Gwak GY, Kim JM, Kwon $\mathrm{CH}$, Joh JW, et al. Steatosis among living liver donors without evidence of fatty liver on ultrasonography: potential implications for preoperative liver biopsy. Transplantation. 2013;95(11):1404-9.

16 Kim NH, Park J, Kim SH, Kim YH, Kim DH, Cho GY, et al. Non-alcoholic fatty liver disease, metabolic syndrome and subclinical cardiovascular changes in the general population. Heart. 2014;100(12):938-43.

17 Younossi ZM, Loomba R, Anstee QM, Rinella ME, Bugianesi E, Marchesini G, et al. Diagnostic modalities for nonalcoholic fatty liver disease, nonalcoholic steatohepatitis, and associated fibrosis. Hepatology. 2018;68(1): $349-60$.

18 Zou B, Yeo YH, Nguyen VH, Cheung R, Ingelsson E, Nguyen MH. Prevalence, characteristics and mortality outcomes of obese, nonobese and lean NAFLD in the United States, 1999-2016. J Intern Med. 2020;288(1): 139-51.

19 Wu Y, Zheng Q, Zou B, Yeo Y, Li X, Li J, et al. The epidemiology of NAFLD in Mainland China with analysis by adjusted gross regional domestic product: a meta-analysis. Hepatol Int. 2020 Mar;14(2):259-69.

20 Akshintala D, Chugh R, Amer F, Cusi K. Nonalcoholic fatty liver disease: the overlooked complication of type 2 diabetes. In: Feingold KR, Anawalt B, Boyce A, Chrousos G, de Herder WW, Dungan K, editors. Endotext. South Dartmouth, MA: MDText.com, Inc.; 2000.

21 Paik JM, Deshpande R, Golabi P, Younossi I, Henry L, Younossi ZM. The impact of modifiable risk factors on the long-term outcomes of non-alcoholic fatty liver disease. Aliment Pharmacol Ther. 2020;51(2):291-304.

22 Targher G, Byrne CD, Tilg H. NAFLD and increased risk of cardiovascular disease: clinical associations, pathophysiological mechanisms and pharmacological implications. Gut. 2020;69(9):1691-705.
23 Golabi P, Fukui N, Paik J, Sayiner M, Mishra A, Younossi ZM. Mortality risk detected by atherosclerotic cardiovascular disease score in patients with nonalcoholic fatty liver disease. Hepatol Commun. 2019 Jun 6;3(8): 1050-60. Available from:

24 Khalid YS, Dasu NR, Suga H, Dasu KN, Reja $\mathrm{D}$, Shah A, et al. Increased cardiovascular events and mortality in females with NAFLD: a meta-analysis. Am J Cardiovasc Dis. 2020; 10(3):258-71.

25 Younossi ZM, Corey KE, Lim JK. AGA clinical practice update on lifestyle modification using diet and exercise to achieve weight loss in the management of nonalcoholic fatty liver disease: expert review. Gastroenterology. 2021 Feb;160(3):912-8.

26 Dulai PS, Singh S, Patel J, Soni M, Prokop LJ, Younossi Z, et al. Increased risk of mortality by fibrosis stage in nonalcoholic fatty liver disease: systematic review and meta-analysis. Hepatology. 2017;65(5):1557-65.

27 Stefan N, Häring HU, Cusi K. Non-alcoholic fatty liver disease: causes, diagnosis, cardiometabolic consequences, and treatment strategies. Lancet Diabetes Endocrinol. 2019;7(4): 313-24.

28 Hazlehurst JM, Woods C, Marjot T, Cobbold JF, Tomlinson JW. Non-alcoholic fatty liver disease and diabetes. Metab Clin Exp. 2016; 65(8):1096-108.

29 Sumida Y, Yoneda M, Tokushige K, Kawanaka M, Fujii H, Yoneda M, et al. Antidiabetic therapy in the treatment of nonalcoholic steatohepatitis. Int J Mol Sci. 2020;21(6):1907.

30 Taylor RS, Taylor RJ, Bayliss S, Hagström H, Nasr P, Schattenberg JM, et al. Association between fibrosis stage and outcomes of patients with nonalcoholic fatty liver disease: a systematic review and meta-analysis. Gastroenterology. 2020;158(6):1611-12.e12.

31 Younossi ZM, Noureddin M, Bernstein D, Kwo P, Russo M, Shiffman ML, et al. Role of noninvasive tests in clinical gastroenterology practices to identify patients with nonalcoholic steatohepatitis at high risk of adverse outcomes: expert panel recommendations. Am J Gastroenterol. 2021 Feb;116(2):254-62.

32 Xiao G, Zhu S, Xiao X, Yan L, Yang J, Wu G. Comparison of laboratory tests, ultrasound, or magnetic resonance elastography to detect fibrosis in patients with nonalcoholic fatty liver disease: a meta-analysis. Hepatology. 2017; 66(5):1486-501.
33 Castera L, Friedrich-Rust M, Loomba R. Noninvasive assessment of liver disease in patients with nonalcoholic fatty liver disease. Gastroenterology. 2019;156(5):1264-81.e4.

34 Eddowes PJ, Sasso M, Allison M, Tsochatzis E, Anstee QM, Sheridan D, et al. Accuracy of fibroscan controlled attenuation parameter and liver stiffness measurement in assessing steatosis and fibrosis in patients with nonalcoholic fatty liver disease. Gastroenterology. 2019;156(6):1717-30.

35 Total population in South Korea from 2014 to 2024: statista [updated 2020 Feb 6]. Available from: https: //www.statista.com/statistics/263747/total-population-in-south-korea/.

36 Korea S. 2017 Population and Housing Census Statistics Korea. 2018. Available from: http: //kostat.go.kr/portal/eng/pressReleases / 1 / index.board ? b mode = read \& aSeq=370994.

37 Cai J, Zhang XJ, Ji YX, Zhang P, She ZG, Li H. Nonalcoholic fatty liver disease pandemic fuels the upsurge in cardiovascular diseases. Circ Res. 2020;126(5):679-704.

38 Bernstein RS, Meurer LN, Plumb EJ, Jackson JL. Diabetes and hypertension prevalence in homeless adults in the United States: a systematic review and meta-analysis. Am J Public Health. 2015;105(2):e46-60.

39 Ballestri S, Capitelli M, Fontana MC, Arioli D, Romagnoli E, Graziosi C, et al. Direct oral anticoagulants in patients with liver disease in the era of non-alcoholic fatty liver disease global epidemic: a narrative review. Adv Ther. 2020;37(5):1910-32.

40 Cheungpasitporn W, Thongprayoon $\mathrm{C}$, Wijarnpreecha K, Mitema DG, Mao MA, Nissaisorakarn $\mathrm{P}$, et al. Decline in prevalence and risk of Helicobacter pylori in kidney transplant recipients: a systematic review and meta-analysis. J Evid Based Med. 2017;10(3): 171-6.

41 Correll CU, Solmi M, Veronese N, Bortolato B, Rosson S, Santonastaso P, et al. Prevalence, incidence and mortality from cardiovascular disease in patients with pooled and specific severe mental illness: a large-scale meta-analysis of $3,211,768$ patients and $113,383,368$ controls. World Psychiatry. 2017;16(2):16380.

42 Younossi ZM, Blissett D, Blissett R, Henry L, Stepanova M, Younossi Y, et al. The economic and clinical burden of nonalcoholic fatty liver disease in the United States and Europe. Hepatology. 2016;64(5):1577-86. 